\title{
COVID-19-related Multisystem Inflammatory Syndrome in a Young Adult
}

\author{
Francisco Pombo, Carolina Seabra, Vergilio Soares, Ana João Sá, Inês Ferreira, Marina Mendes \\ Centro Hospitalar do Tâmega e Sousa, Penafiel, Portugal
}

Doi: 10.12890/2021_002520- European Journal of Case Reports in Internal Medicine - ๔ EFIM 2021

Received: $26 / 03 / 2021$

Accepted: 30/03/2021

Published: $19 / 04 / 2021$

How to cite this article: Pombo F, Seabra C, Soares V, Sá AJ, Ferreira I, Mendes M. COVID-19-related multisystem inflammatory syndrome in a young adult. EJCRIM 2021;8: doi:10.12890/2021_002520.

Conflicts of Interests: The Authors declare that there are no competing interests.

This article is licensed under a Commons Attribution Non-Commercial 4.0 License

\section{ABSTRACT}

Introduction: A condition called multisystem inflammatory syndrome in children (MIS-C), related to past SARS-CoV-2 infection, has been described in a series of cases. There is a growing body of evidence suggesting a similar entity in adults (MIS-A). We report a case of a young adult with a hyperinflammatory systemic syndrome with end-organ lesions and a recent SARS-CoV-2 infection.

Methods and Procedures: The patient developed end-organ lesions in the respiratory, cardiovascular, digestive, haematological and mucocutaneous systems. A wide diagnostic work-up did not find any specific aetiology (autoimmunity, neoplastic or infectious). There was evidence of past SARS-CoV-2 infection with positive autoimmune titres (positive IgG). A presumptive diagnosis of MIS-A was made and treatment with corticosteroids was introduced with improvement.

Discussion: COVID-19-related multisystem inflammatory syndrome in adults is still an unknown entity with no diagnostic criteria or treatment guidelines. Much of what is known is inferred from what we already know about MIS-C. There have been several reported cases with severity ranging from mild to severe. The mechanisms behind this condition are still largely unknown. More evidence is needed to establish diagnosis and treatment.

\section{LEARNING POINTS}

- COVID-19-related multisystem inflammatory syndrome in adults is a new entity with few case reports.

- Awareness is important for early diagnosis and treatment

\section{KEYWORDS}

COVID-19, multisystem inflammatory syndrome, adult.

\section{INTRODUCTION}

Since the beginning of the SARS-CoV-2 pandemic, a series of Kawasaki disease-like cases in children has been described. An entity called multisystem inflammatory syndrome in children (MIS-C), related to past SARS-CoV-2 infection, has begun to get broader attention. This has a different set of features from Kawasaki disease, affecting older children and a wider range of organs. Severity varies from mild to severe. A series of multisystem inflammatory syndrome in adults (MIS-A) has also been described, raising awareness of this syndrome.

MIS-A is a multisystem inflammatory syndrome affecting a wide range of organs in younger adults (most cases were in patients less than 50 years old), with severity varying from mild to severe disease or even death, mainly from cardiogenic shock. It presents with elevated inflammation markers with end-organ lesions in patients with current or past (confirmed with antibody titres) SARS-CoV-2 infection. We describe the case of a 24-year-old man of European origin, without a relevant medical history but with SARS-CoV-2 infection diagnosed 1 month previously. He presented to the emergency department with fever, diarrhoea and abdominal pain for the past 3 days. 
He had normal blood pressure (123/58 $\mathrm{mmHg}$ ), heart rate $(93 \mathrm{bpm})$ and peripheral saturation (100\%), and fever $\left(39^{\circ} \mathrm{C}\right)$. Abdominal palpation revealed acute pain in the lower right abdominal quadrant without any signs of peritoneal irritation. Laboratory findings showed mild leucocytosis $\left(12 \times 10^{\%} / \mathrm{I}\right)$ and neutrophilia ( $\left.85 \%\right)$, thrombocytosis $\left(459 \times 10^{\%} / \mathrm{I}\right)$ and an elevated C-reactive protein (418.9 mg/l). An ultrasound showed thickening of the ileum with a small peritoneal effusion seen on a CT scan. The patient was hospitalized in the internal medicine department given the diagnostic uncertainty and no evidence of a surgical indication, such as appendicitis.

\section{METHODS AND PROCEDURES}

A colonoscopy was performed which showed no signs of inflammatory bowel disease. On the 4th day of hospitalization, the patient's symptoms worsened with persistent fever, cough, hypotension, dyspnoea and cutaneous lesions. These were macular, erythematous, multiforme, non-itching or painful, and dispersed over the thorax, abdomen, arms and legs. Mild type 1 respiratory failure ( $\left.\mathrm{PaO}_{2} / \mathrm{FiO}_{2} 261\right)$ was noted. Laboratory findings revealed a systemic hyperinflammatory state (elevated C-reactive protein, fibrinogen, ferritin, D-dimers, lactate dehydrogenase and a prolonged sedimentation rate), elevation of high-sensitivity troponin I and NT-proBNP, prolonged INR and normal renal, liver and thyroid functions (Table 1). There were no findings on the electrocardiogram. Thoracic CT angiography showed the presence of ground-glass opacities, small pericardial and pleural effusions and no evidence of pulmonary embolism. Transthoracic echocardiography exhibited a left ventricular ejection fraction in the lower normal range, with no other abnormalities.

\begin{tabular}{|c|c|c|}
\hline & At admission & At discharge \\
\hline Haemoglobin (g/dl) & 11.4 & 11.9 \\
\hline White blood count $\left(2-8 \times 10^{9} / I\right)$ & 12.9 & 7.83 \\
\hline Neutrophil count (\%) & 79.6 & 50.2 \\
\hline Lymphocyte count (\%) & 9.0 & 38.8 \\
\hline Eosinophil count (\%) & 7.6 & 0.9 \\
\hline 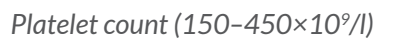 & 469 & 747 \\
\hline INR & 1.44 & 1.16 \\
\hline Creatinine (mg/dl) & 0.74 & 0.85 \\
\hline Urea (mg/dl) & 17 & 45 \\
\hline Sodium (mmol/l) & 139 & 137 \\
\hline Potassium (mmol/l) & 3.7 & 4.4 \\
\hline Albumin (g/dl) & 2.8 & 3.3 \\
\hline High-sensitivity troponin I ( $\mathrm{gg} / \mathrm{ml})$ & 518 & 30.8 \\
\hline NT-proBNP (pg/ml) & 10,900 & NM \\
\hline C-reactive protein $(\mathrm{mg} / \mathrm{l})$ & 418.9 & 13.9 \\
\hline Sedimentation rate (seconds) & 102 & NM \\
\hline Fibrinogen (mg/dl) & 1015 & 334 \\
\hline$D$-dimers (ng/ml) & 1400 & 358 \\
\hline Ferritin (ng/ml) & 1366 & 432 \\
\hline Lactate dehydrogenase (U/I) & 239 & 177 \\
\hline Haptoglobin (mg/dl) & 316 & NM \\
\hline Faecal calprotectin $(\mu \mathrm{g} / \mathrm{g})$ & 31 & NM \\
\hline
\end{tabular}

Table 1. Laboratory assessment at admission and discharge. I NR, international normalized ratio; NM, not measured 


\section{of Case Reports in \\ Internal Medicine}

The diagnostic work-up included a wide range of autoantibodies, viral and bacterial serologies and haemocultures, which were all negative (Table 2). Antibodies for SARS-CoV-2 confirmed prior infection (negative IgM and positive IgG). Full-body CT displayed no evidence of neoplastic disease.

\begin{tabular}{|l|c|}
\hline & Results \\
\hline ANA & N \\
\hline Anti-dsDNA & N \\
\hline ANCA & N \\
\hline Anti-GBM & N \\
\hline Rheumatoid factor & N \\
\hline Anti-CCP & 95 \\
\hline Complement C3 (mg/dl) & 5 \\
\hline Complement C4 (mg/dl) & $N$ \\
\hline Faecal microbiology & $N$ \\
\hline Serum microbiology & $N$ \\
\hline HIV 1 and 2 & $N$ \\
\hline HBV and HCV & $N$ \\
\hline Adenovirus & $N$ \\
\hline Coxsackie A9, B1-6 & \\
\hline
\end{tabular}

\begin{tabular}{|l|c|}
\hline & Results \\
\hline Echovirus & $N$ \\
\hline HHV6 & $N$ \\
\hline Parvovirus B19 & IgM N; IgG P \\
\hline Influenza A, B & $N$ \\
\hline Parainfluenza 1,2,3 & $N$ \\
\hline Varicella-zoster & IgM N; IgGP \\
\hline SARS-CoV-2 & IgM N; IgGP \\
\hline Leptospira & $N$ \\
\hline Bartonella sp. & $N$ \\
\hline Coxiella burnetii & $N$ \\
\hline Borrelia burgdorferi & $N$ \\
\hline Rickettsia conorii & $N$ \\
\hline Rickettsia typhi & $N$ \\
\hline Haemophilus influenzae & IgM N; IgG P \\
\hline
\end{tabular}

Table 2. Diagnostic work-up. $N$, negative; $P$, positive

Since the patient showed signs of a multisystem inflammatory condition with end-organ damage (respiratory, cardiovascular, cutaneous, haematological and intestinal), treatment with systemic corticosteroids (first intravenous methylprednisolone $32 \mathrm{mg} / \mathrm{day}$ and then oral prednisolone $40 \mathrm{mg} / \mathrm{day}$ ) was begun, with dramatic clinical and laboratory improvement. After 1 week, the cutaneous lesions had resolved and inflammatory markers were within normal ranges. A second CT scan showed no signs of ground-glass opacities, pericardial, pleural or peritoneal effusion or intestinal thickening, while a second transthoracic echocardiogram demonstrated a normal left ventricular ejection fraction. The patient was discharged on slowly tapering prednisolone with no symptom recurrence in the following 4 weeks.

As the diagnostic work-up was inconclusive, and there were no other plausible causes, and the patient had a confirmed previous SARSCoV-2 infection, we consider this to be a case of SARS-CoV-2-related multisystem inflammatory syndrome in adults.

\section{DISCUSSION}

The multisystem inflammatory syndrome in adults is a new entity with little literature supporting its diagnostic work-up and treatment. Much of what is known is inferred from what is already known about MIS-C. However, both the World Health Organization ${ }^{[1]}$ and the Center for Disease Control and Prevention ${ }^{[2]}$ have published guidelines on the case definition of MIS-C. If we take these into account, our patient met all criteria in both definitions, except for the age criteria.

From cases that have already been reported in the medical literature, it is seen that MIS-A has a wide range of clinical manifestations, varying from mild to severe ${ }^{[3]}$. Chau et al. report a series of seven males, ranging in age from 20 to 42 years old, with positive SARS-CoV-2 antibodies and a hyperinflammatory state which presented as cardiogenic shock and showed improvement after treatment with corticosteroids ${ }^{[4]}$. In fact, as in Kawasaki disease in children, a medium-sized artery vasculitis with systemic manifestations, which seems to be related to an unknown infectious agent ${ }^{[5]}$, and in MIS-C, in which children had prior SARS-CoV-2 infection, MIS-A seems to be a hyperinflammatory reaction with end-organ lesions after infection. The mechanisms behind these are still unknown. Thrombotic events and coagulopathy have been reported in these patients, suggesting a relationship between the hyperinflammatory and hypercoagulable states ${ }^{[6]}$. 


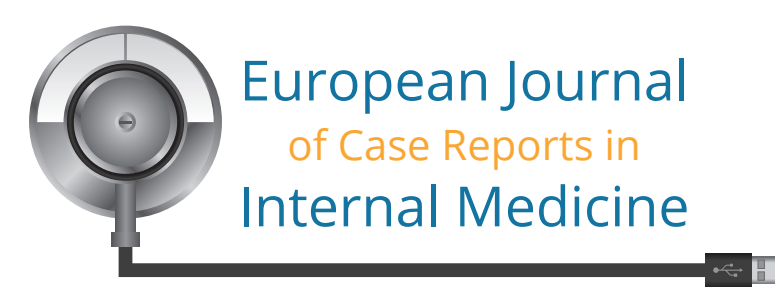

Complement deposition on microvasculature seems to play a role in this relationship, with deposition of complement components in the lung and skin lesions reported by Magro et al. ${ }^{[7]}$.

Despite these findings and the growing body of knowledge about MIS-C, MIS-A is still relatively unknown, making it a necessity to establish diagnostic criteria and treatment guidelines to better guide clinicians.

\section{REFERENCES}

1. World Health Organization. Multisystem inflammatory syndrome in children and adolescents temporally related to COVID-19. 15 May 2020 Scientific Brief. Geneva: World Health Organization; 2020.

2. Centers for Disease Control and Prevention. Multisystem Inflammatory Syndrome in Children (MIS-C) associated with Coronavirus Disease 2019 (COVID-19). May 2020. Atlanta, GA: U.S. Department of Health and Human Services, Centers for Disease Control and Prevention; 2020.

3. Kaushik S, Aydin SI, Derespina KR, Bansal PB, Kowalsky S, Trachtman R, et al. Multisystem inflammatory syndrome in children associated with severe acute respiratory syndrome coronavirus 2 infection (MIS-C): a multi-institutional study from New York City. J Pediatr 2020;224:24-29.

4. Chau VQ, Giustino G, Mahmood K, Oliveros E, Neibart E, Oloomi M, et al. Cardiogenic shock and hyperinflammatory syndrome in young males with COVID-19. Circ Heart Fail 2020;13(10):e007485.

5. Nakamura Y. Kawasaki disease: epidemiology and the lessons from it. Int J Rheum Dis 2018;21(1):16-19.

6. Colling Meaghan E, Kanthi Y. COVID-19-associated coagulopathy: an exploration of mechanisms. Vasc Med 2020;25(5):471-478

7. Magro C. Complement associated microvascular injury and thrombosis in the pathogenesis of severe COVID-19 infection: a report of five cases. TransI Res 2020;220:1-13. 